\title{
Identifying weak focus of order 3 in a Leslie-Gower prey-predator model with prey harvesting
}

\section{Juan $\mathrm{Su}^{1 *}$ (D)}

\section{"Correspondence:}

sujuanmath@163.com

'Department of Mathematics, Chengdu Normal University,

Chengdu, China

\section{Springer}

\begin{abstract}
In this paper, we identify a weak focus with order up to 3 for a Leslie-Gower prey-predator model with three parameters. The known work computed the first Lyapunov quantity and discussed Hopf bifurcations of this system, but the identification of weak focus and its maximal order was not completed yet. In this paper, we decompose the varieties of the first three Lyapunov quantities of this system by resultant, realroot isolation, and pseudo-division so as to prove that the center-type equilibrium is a weak focus with order up to 3 . Moreover, we give the parameter conditions of each order. Finally, numerical simulations are employed to illustrate the results obtained.
\end{abstract}

MSC: $34 C 25 ; 92 D 25$

Keywords: Leslie-Gower prey-predator model; Weak focus; Lyapunov quantity; Resultant; Realroot isolation

\section{Introduction}

Studies on dynamics of prey-predator models give better understanding of the relations between two species, and provide management for sustainable development. One of the important models is the Leslie-Gower prey-predator model $[21,22]$ given by

$$
\left\{\begin{array}{l}
\dot{x}=r_{1} x\left(1-\frac{x}{K}\right)-y p(x), \\
\dot{y}=r_{2} y\left(1-\frac{y}{b x}\right)
\end{array}\right.
$$

where $x(t)$ and $y(t)$ are densities of prey and predator at time $t$, respectively. The prey grows with intrinsic growth rate $r_{1}$ and carrying capacity $K$ in the absence of predation. Functional response function $p(x)$ describes the feeding rate of prey consumption by predators. The predator, according to the numerical response of Leslie-Gower type [18, 19], grows with intrinsic growth rate $r_{2}$ and carrying capacity $b x$ proportional to the population of prey, where $b$ is a measure of the food quality of prey for conversion into predator births. Moreover, $r_{1}, r_{2}, K$, and $b$ are all positive constants.

Dynamics of system (1.1) has been studied extensively when the functional response $p(x)$ is of Holling types. Hsu and Huang [10] investigated the global stability of system (1.1) with $p(x)$ being Holling types I, II, and III. Moreover, they $[11,12]$ studied the limit

(c) The Author(s) 2019. This article is distributed under the terms of the Creative Commons Attribution 4.0 International License (http://creativecommons.org/licenses/by/4.0/), which permits unrestricted use, distribution, and reproduction in any medium, provided you give appropriate credit to the original author(s) and the source, provide a link to the Creative Commons license, and indicate if changes were made. 
cycles and Hopf bifurcation of this system when $p(x)$ is type II. Huang, Ruan, and Song [15] studied the local bifurcations of system (1.1) when $p(x)$ is Holling type III. Li and Xiao [20] and Huang et al. [16] investigated the bifurcations of this system with $p(x)$ being Holling type IV.

Harvesting is commonly practiced in fishery, forestry, and wildlife management. It is very important to harvest biological resources with maximum sustainable yield while maintaining the survival of all interacting population. Recently, much attention has been paid to the dynamics of prey-predator model with harvesting (see, e.g., $[1-3,7,13,14,24$, $25])$. When $p(x)=a x$, i.e., Holling type I, Zhu and Lan in [27] studied system (1.1) with constant harvest $h$ on prey, i.e.,

$$
\left\{\begin{array}{l}
\dot{x}=r_{1} x\left(1-\frac{x}{K}\right)-a x y-h, \\
\dot{y}=r_{2} y\left(1-\frac{y}{b x}\right),
\end{array}\right.
$$

where $a>0$ is a constant and $h>0$. By the rescaling $x \rightarrow x / K, y \rightarrow a y / r_{1}$ and $t \rightarrow r_{1} t$ as in [27], system (1.2) reads

$$
\left\{\begin{array}{l}
\dot{x}=x(1-x)-x y-\varepsilon, \\
\dot{y}=y\left(\delta-\frac{\beta y}{x}\right)
\end{array}\right.
$$

where $\beta=r_{2} /(a b K), \delta=r_{2} / r_{1}$, and $\varepsilon=h /\left(r_{1} K\right)$. It was proved in [8,27] that system (1.3) may undergo a saddle-node bifurcation and a Bogdanov-Takens bifurcation with codimension 2. Moreover, Hopf bifurcations of this system were also studied in [27, Theorem 4.3] when the first Lyapunov number does not vanish. However, there is no further discussion about the identification of weak focus or center when the first Lyapunov number vanishes.

In this paper, we continue the identification of weak focus or center in system (1.3). By Lyapunov numbers, we prove that the equilibrium of center type in this system is a weak focus with order up to 3 and can be exactly. The main difficulty comes from the computation of zeros of Lyapunov numbers restricted to subsets of biological sense. Such problem is solved by resultant [17], pseudo-division [23], and realroot isolation [6]. Moreover, parameter conditions of each order are given. Simulation of two limit cycles arisen from a degenerate Hopf bifurcation is employed to illustrate our results.

\section{Condition of center type}

For the biological sense, we discuss system $(1.3)$ in the region $(0,+\infty) \times[0,+\infty)$ as in $[8$, 27]. The following lemma gives the number of equilibria of system (1.3).

Lemma 2.1 ([27, Theorem 3.1]) Let $\beta, \delta$, and $\varepsilon>0$.

(i) If $\varepsilon>\frac{1}{4}$, then system (1.3) has no equilibrium.

(ii) If $\varepsilon=\frac{1}{4}$, then system (1.3) has only one equilibrium $\left(\frac{1}{2}, 0\right)$.

(iii) If $\frac{\beta}{4(\delta+\beta)}<\varepsilon<\frac{1}{4}$, then system (1.3) has exactly two equilibria $\left(x_{1}, 0\right)$ and $\left(x_{2}, 0\right)$, where $x_{1}=\frac{1}{2}(1-\sqrt{1-4 \varepsilon})$ and $x_{2}=\frac{1}{2}(1+\sqrt{1-4 \varepsilon})$.

(iv) If $\varepsilon=\frac{\beta}{4(\delta+\beta)}$, then system (1.3) has three equilibria $\left(x_{1}, 0\right),\left(x_{2}, 0\right)$, and $\left(\frac{\beta}{2(\delta+\beta)}, \frac{\delta}{2(\delta+\beta)}\right)$.

(v) If $\varepsilon<\frac{\beta}{4(\delta+\beta)}$, then system (1.3) has four equilibria $\left(x_{1}, 0\right),\left(x_{2}, 0\right),\left(x_{3}, y_{3}\right)$, and $\left(x_{4}, y_{4}\right)$, where $x_{3}=\frac{\beta-\sqrt{-4 \beta \delta \varepsilon-4 \beta^{2} \varepsilon+\beta^{2}}}{2(\delta+\beta)}, x_{4}=\frac{\beta+\sqrt{-4 \beta \delta \varepsilon-4 \beta^{2} \varepsilon+\beta^{2}}}{2(\delta+\beta)}$, and $y_{i}=\frac{\delta x_{i}}{\beta}, i=3,4$. 
Table 1 Properties of $\left(x_{4}, y_{4}\right)$

\begin{tabular}{llll}
\hline Parameter conditions & & Properties \\
\hline $0<\beta<\frac{1}{2}$ & $0<\delta<\frac{1}{2}-\beta$ & $0<\varepsilon<\varepsilon_{1}$ & stable node or focus \\
& & & center type \\
& $\varepsilon_{1}<\varepsilon<\frac{\beta}{4(\beta+\delta)}$ & unstable node or focus \\
$0<\beta<\frac{1}{2}$ & $\delta \geq \frac{1}{2}-\beta$ & $0<\varepsilon<\frac{\beta}{4(\beta+\delta)}$ & stable node or focus \\
$\beta \geq \frac{1}{2}$ & $\delta>0$ & $0<\varepsilon<\frac{\beta}{4(\beta+\delta)}$ & stable node or focus \\
\hline
\end{tabular}

The dynamics of all equilibria above was discussed in [27, Theorem 3.3, 3.4, 3.5 ]. It shows that only the equilibrium $\left(x_{4}, y_{4}\right)$ can be of center type for certain parameter values. Let

$$
\varepsilon_{1}=\frac{\beta}{4(\beta+\delta)}\left(1-\left(\frac{\delta-2 \delta(\beta+\delta)}{2 \beta+\delta}\right)^{2}\right)
$$

Then the following lemma gives the qualitative properties of $\left(x_{4}, y_{4}\right)$.

Lemma 2.2 ([27, Theorem 3.4]) Dynamics of $\left(x_{4}, y_{4}\right)$ are given in Table 1.

For convenience, let

$$
\Lambda:=\left\{(\beta, \delta, \varepsilon) \in \mathbb{R}_{3}: 0<\beta<\frac{1}{2}, 0<\delta<\frac{1}{2}-\beta, \varepsilon=\varepsilon_{1}\right\} .
$$

Then, for $(\beta, \delta, \varepsilon) \in \Lambda,\left(x_{4}, y_{4}\right)=\left(\frac{\beta(1-\delta)}{2 \beta+\delta}, \frac{\delta(1-\delta)}{2 \beta+\delta}\right)$, and the Jacobian matrix of system (1.3) at this point is given by

$$
J\left(x_{4}, y_{4}\right)=\left[\begin{array}{cc}
\delta & -\frac{\beta(1-\delta)}{2 \beta+\delta} \\
\frac{\delta^{2}}{\beta} & -\delta
\end{array}\right]
$$

So $J\left(x_{4}, y_{4}\right)$ has a pair of imaginary eigenvalues $\mathbf{i} \delta \sqrt{\frac{1-2(\beta+\delta)}{2 \beta+\delta}}$. Moreover, by Theorem 4.3 in [27], the subcritical and supercritical Hopf bifurcations were discussed when the first Lyapunov number does not vanish. However, there is no further discussion when the Lyapunov number vanishes.

\section{Identification of weak focus}

In this section, we complete the identification of weak focus for $\left(x_{4}, y_{4}\right)$ for all $(\beta, \delta, \varepsilon) \in \Lambda$.

Theorem 3.1 Let $(\beta, \delta, \varepsilon) \in \Lambda$. Then $\left(x_{4}, y_{4}\right)=\left(\frac{\beta(1-\delta)}{2 \beta+\delta}, \frac{\delta(1-\delta)}{2 \beta+\delta}\right)$ is a weak focus of order at most 3 .

To make the preparation, we compute the first three Lyapunov numbers of this system. By the translation $x \rightarrow x-x_{4}, y \rightarrow y-y_{4}$ and Taylor expansions, system (1.3) be- 
comes

$$
\left\{\begin{aligned}
\dot{x}= & \delta x+\frac{\beta(\delta-1)}{2 \beta+\delta} y-x^{2}-x y, \\
\dot{y}= & \frac{\delta^{2}}{\beta} x-\delta y+\frac{\delta^{2}(2 \beta+\delta)}{\beta^{2}(\delta-1)} x^{2}-\frac{2 \delta(2 \beta+\delta)}{\beta(\delta-1)} x y+\frac{(2 \beta+\delta)}{\delta-1} y^{2}+\frac{\delta^{2}(2 \beta+\delta)^{2}}{\beta^{3}(\delta-1)^{2}} x^{3}-\frac{2 \delta(2 \beta+\delta)^{2}}{\beta^{2}(\delta-1)^{2}} x^{2} y \\
& +\frac{(2 \beta+\delta)^{2}}{(\delta-1)^{2} \beta} x y^{2}+\frac{\delta^{2}(2 \beta+\delta)^{3}}{\beta^{4}(\delta-1)^{3}} x^{4}-\frac{2 \delta(2 \beta+\delta)^{3}}{\beta^{3}(\delta-1)^{3}} x^{3} y+\frac{(2 \beta+\delta)^{3}}{\beta^{2}(\delta-1)^{3}} x^{2} y^{2}+\frac{\delta^{2}(2 \beta+\delta)^{4}}{\beta^{5}(\delta-1)^{4}} x^{5} \\
& -\frac{2 \delta(2 \beta+\delta)^{4}}{\beta^{4}(\delta-1)^{4}} x^{4} y+\frac{(2 \beta+\delta)^{4}}{\beta^{3}(\delta-1)^{4}} x^{3} y^{2}+\frac{\delta^{2}(2 \beta+\delta)^{5}}{\beta^{6}(\delta-1)^{5}} x^{6}-\frac{2 \delta(2 \beta+\delta)^{5}}{\beta^{5}(\delta-1)^{5}} x^{5} y+\frac{(2 \beta+\delta)^{5}}{\beta^{4}(\delta-1)^{5}} x^{4} y^{2} \\
& +\frac{\delta^{2}(2 \beta+\delta)^{6}}{\beta^{7}(\delta-1)^{6}} x^{7}-\frac{2 \delta(2 \beta+\delta)^{6}}{\beta^{6}(\delta-1)^{6}} x^{6} y+\frac{(2 \beta+\delta)^{6}}{\beta^{5}(\delta-1)^{6}} x^{5} y^{2}+O\left(|x, y|^{8}\right) .
\end{aligned}\right.
$$

With the change of variables $[9$, Sect. 2.1]

$$
u=\frac{x}{2}, \quad v=-\frac{\sqrt{2 \beta+\delta}}{2 \sqrt{1-2 \beta-2 \delta}} x+\frac{\beta(1-\delta)}{2 \delta \sqrt{(2 \beta+\delta)(1-2 \beta-2 \delta)}} y
$$

and time rescaling $t \rightarrow \delta \sqrt{\frac{1-2(\beta+\delta)}{2 \beta+\delta}} t$, system (3.1) can be written as

$$
\begin{aligned}
& \dot{u}=-v+\frac{2\left(\beta \delta+\delta^{2}+\beta\right)}{\beta \delta(\delta-1) \sqrt{-\frac{2 \delta-1+2 \beta}{2 \beta+\delta}}} u^{2}+\frac{2(2 \beta+\delta)}{\beta(\delta-1)} u v \\
& \dot{v}=u+\frac{2\left(4 \beta^{2} \delta+9 \beta \delta^{2}+5 \delta^{3}-4 \beta \delta-5 \delta^{2}-\beta+\delta\right)(2 \beta+\delta)}{\beta \delta(\delta-1)^{2}(2 \delta-1+2 \beta)} u^{2} \\
& -\frac{2(2 \beta+\delta)(5 \delta-3+4 \beta)}{\beta(\delta-1)^{2} \sqrt{-\frac{2 \delta-1+2 \beta}{2 \beta+\delta}}} u \nu-\frac{2(2 \beta+\delta)^{2}}{(\delta-1)^{2} \beta} v^{2} \\
& +4 \frac{(2 \beta+\delta)^{2}(2 \delta-1+2 \beta)}{\beta^{2}(\delta-1)^{3}} u^{3}+\frac{8(2 \beta+\delta)^{3} \sqrt{-\frac{2 \delta-1+2 \beta}{2 \beta+\delta}}}{\beta^{2}(\delta-1)^{3}} u^{2} v-\frac{4(2 \beta+\delta)^{3}}{\beta^{2}(\delta-1)^{3}} u v^{2} \\
& +\frac{8(2 \beta+\delta)^{3}(2 \delta-1+2 \beta)}{\beta^{3}(\delta-1)^{4}} u^{4}+\frac{16(2 \beta+\delta)^{4} \sqrt{-\frac{2 \delta-1+2 \beta}{2 \beta+\delta}}}{\beta^{3}(\delta-1)^{4}} u^{3} v-\frac{8(2 \beta+\delta)^{4}}{\beta^{3}(\delta-1)^{4}} u^{2} v^{2} \\
& +\frac{16(2 \beta+\delta)^{4}(2 \delta-1+2 \beta)}{\beta^{4}(\delta-1)^{5}} u^{5}+\frac{32(2 \beta+\delta)^{5} \sqrt{-\frac{2 \delta-1+2 \beta}{2 \beta+\delta}}}{\beta^{4}(\delta-1)^{5}} u^{4} v-\frac{16(2 \beta+\delta)^{5}}{\beta^{4}(\delta-1)^{5}} u^{3} v^{2} \\
& +\frac{32(2 \beta+\delta)^{5}(2 \delta-1+2 \beta)}{\beta^{5}(\delta-1)^{6}} u^{6}+\frac{64(2 \beta+\delta)^{6} \sqrt{-\frac{2 \delta-1+2 \beta}{2 \beta+\delta}}}{\beta^{5}(\delta-1)^{6}} u^{5} v-\frac{32(2 \beta+\delta)^{6}}{\beta^{5}(\delta-1)^{6}} u^{4} v^{2} \\
& +\frac{64(2 \beta+\delta)^{6}(2 \delta-1+2 \beta)}{\beta^{6}(\delta-1)^{7}} u^{7}+\frac{128(2 \beta+\delta)^{7} \sqrt{-\frac{2 \delta-1+2 \beta}{2 \beta+\delta}}}{\beta^{6}(\delta-1)^{7}} u^{6} v-\frac{64(2 \beta+\delta)^{7}}{\beta^{6}(\delta-1)^{7}} u^{5} v^{2} \\
& +O\left(|u, v|^{8}\right) \text {. }
\end{aligned}
$$

In the polar coordinate $u=r \cos \theta$ and $v=r \sin \theta$, system (3.2) takes the form

$$
\frac{d r}{d \theta}=R_{2}(\theta) r^{2}+R_{3}(\theta) r^{3}+R_{4}(\theta) r^{4}+R_{5}(\theta) r^{5}+R_{6}(\theta) r^{6}+R_{7}(\theta) r^{7}+O\left(r^{8}\right)
$$

where each $R_{i}, i=2,3, \ldots, 7$, is a polynomial of $\sin \theta$ and $\cos \theta$, and its coefficients are determined by the coefficients of system (3.2). Then we consider solutions of system (3.3) in 
the formal series

$$
r\left(\theta, r_{0}\right)=\sum_{i=1}^{\infty} r_{i}(\theta) r_{0}^{i}
$$

with the initial condition

$$
r\left(0, r_{0}\right)=r_{0}
$$

where $r_{0}>0$ is sufficiently small. Substituting (3.4) into (3.3) and comparing the coefficients, we have

$$
\begin{aligned}
\dot{r}_{2}(\theta)= & R_{2}, \quad \dot{r}_{3}(\theta)=R_{3}+2 R_{2} r_{2}, \quad \dot{r}_{4}(\theta)=R_{4}+3 R_{3} r_{2}+R_{2}\left(r_{2}^{2}+2 r_{3}\right), \\
\dot{r}_{5}(\theta)= & R_{5}+4 R_{4} r_{2}+3 R_{3}\left(r_{2}^{2}+r_{3}\right)+2 R_{2}\left(r_{2} r_{3}+r_{4}\right), \\
\dot{r}_{6}(\theta)= & R_{6}+5 R_{5} r_{2}+\left(6 r_{2}^{2}+4 r_{3}\right) R_{4}+\left(r_{2}^{3}+6 r_{2} r_{3}+3 r_{4}\right) R_{3} \\
& +\left(2 r_{2} r_{4}+r_{3}^{2}+2 r_{5}\right) R_{2}, \\
\dot{r}_{7}(\theta)= & R_{7}+6 r_{2} R_{6}+\left(10 r_{2}^{2}+5 r_{3}\right) R_{5}+\left(4 r_{2}^{3}+12 r_{2} r_{3}+4 r_{4}\right) R_{4} \\
& +\left(3 r_{2}^{2} r_{3}+6 r_{2} r_{4}+3 r_{3}^{2}+3 r_{5}\right) R_{3}+\left(2 r_{2} r_{5}+2 r_{3} r_{4}+2 r_{6}\right) R_{2} .
\end{aligned}
$$

Initial condition (3.5) is equivalent to

$$
r_{1}(0)=1, \quad r_{2}(0)=r_{3}(0)=\cdots=0 \text {. }
$$

By (3.6) and (3.7), we compute the first three Lyapunov numbers $[9,26]$ and obtain

$$
\begin{aligned}
& L_{1}:=\frac{1}{2 \pi} r_{3}(2 \pi)=-\frac{(2 \beta+\delta) f_{1}(\beta, \delta)}{2 \beta^{2} \delta^{2}(1-\delta)^{2} \sqrt{\frac{1-2(\beta+\delta)}{2 \beta+\delta}}}, \\
& L_{2}:=\frac{1}{2 \pi} r_{5}(2 \pi)=-\frac{(2 \beta+\delta)^{2} f_{2}(\beta, \delta)}{1440 \delta^{4} \beta^{4}(1-\delta)^{6}(1-2(\delta+\beta))^{3} \sqrt{\frac{1-2(\beta+\delta)}{2 \beta+\delta}}}, \\
& L_{3}:=\frac{1}{2 \pi} r_{7}(2 \pi)=-\frac{(2 \beta+\delta)^{3} f_{3}(\beta, \delta)}{290,304 \beta^{6} \delta^{6}(1-\delta)^{10}(1-2(\beta+\delta))^{5} \sqrt{\frac{1-2(\beta+\delta)}{2 \beta+\delta}}},
\end{aligned}
$$

where

$$
\begin{aligned}
f_{1}= & -\delta^{4}+\beta \delta^{3}+\left(2 \beta^{2}-6 \beta\right) \delta^{2}+\left(-4 \beta^{2}+\beta\right) \delta-2 \beta^{2}, \\
f_{2}= & 44,032 \beta^{7} \delta^{4}+159,744 \beta^{6} \delta^{5}+213,160 \beta^{5} \delta^{6}+113,134 \beta^{4} \delta^{7}-7311 \beta^{3} \delta^{8} \\
& -38,891 \beta^{2} \delta^{9}-18,873 \beta \delta^{10}-2979 \delta^{11}-88,064 \beta^{7} \delta^{3}-498,688 \beta^{6} \delta^{4} \\
& -1,046,432 \beta^{5} \delta^{5}-1,105,866 \beta^{4} \delta^{6}-647,821 \beta^{3} \delta^{7}-193,579 \beta^{2} \delta^{8}-12,702 \beta \delta^{9} \\
& +3114 \delta^{10}-44,032 \beta^{7} \delta^{2}+37,888 \beta^{6} \delta^{3}+511,528 \beta^{5} \delta^{4}+938,282 \beta^{4} \delta^{5} \\
& +815,517 \beta^{3} \delta^{6}+363,446 \beta^{2} \delta^{7}+55,850 \beta \delta^{8}-175 \delta^{9}+25,600 \beta^{6} \delta^{2}-85,024 \beta^{5} \delta^{3} \\
& -318,382 \beta^{4} \delta^{4}-407,661 \beta^{3} \delta^{5}-258,846 \beta^{2} \delta^{6}-47,730 \beta \delta^{7}-628 \delta^{8}-32,768 \beta^{6} \delta
\end{aligned}
$$




$$
\begin{aligned}
& -57,224 \delta^{2} \beta^{5}-7774 \beta^{4} \delta^{3}+60,035 \beta^{3} \delta^{4}+93,521 \beta^{2} \delta^{5}+18,575 \beta \delta^{6}-20 \delta^{7} \\
& +9472 \beta^{5} \delta+3050 \delta^{2} \beta^{4}+5937 \beta^{3} \delta^{3}-24,775 \beta^{2} \delta^{4}-5252 \beta \delta^{5}-7816 \beta^{5}-6042 \beta^{4} \delta \\
& -12,177 \beta^{3} \delta^{2}+1332 \beta^{2} \delta^{3}+500 \beta \delta^{4}-1682 \beta^{4}+841 \beta^{3} \delta
\end{aligned}
$$

and $f_{3}$ is given in the Appendix.

Let $V\left(\xi_{1}, \xi_{2}, \ldots, \xi_{n}\right)$ be the set of common zeros of $\xi_{i}, i=1,2, \ldots, n$, and denote

$$
\mathcal{D}:=\left\{(\beta, \delta) \in \mathbb{R}_{2}: 0<\beta<\frac{1}{2}, 0<\delta<\frac{1}{2}-\beta\right\} .
$$

Then we have the following lemma which is useful in the proof of Theorem 3.1.

Lemma 3.1 $V\left(f_{1}, f_{2}, f_{3}\right) \cap \mathcal{D}=\emptyset$.

Proof To simplify the set $V\left(f_{1}, f_{2}, f_{3}\right)$, we calculate the resultants [17] by Maple and obtain

$$
\begin{aligned}
& r_{12}:=\operatorname{res}\left(f_{1}, f_{2}, \delta\right)=849,346,560,000 \beta^{12}(2 \beta+1)^{16} \mathcal{R}_{1}, \\
& r_{13}:=\operatorname{res}\left(f_{1}, f_{3}, \delta\right)=110,075,314,176 \beta^{16}(2 \beta+1)^{26} \mathcal{R}_{2}, \\
& r_{23}:=\operatorname{res}\left(r_{12}, r_{13}, \beta\right)=0,
\end{aligned}
$$

where

$$
\begin{aligned}
\mathcal{R}_{1}= & 32 \beta^{3}+96 \beta^{2}-354 \beta+3 \\
\mathcal{R}_{2}= & 104,783,189,114,880,000 \beta^{12}+137,477,299,942,195,200 \beta^{11} \\
& -4,012,954,143,001,436,160 \beta^{10}+22,669,901,410,243,203,072 \beta^{9} \\
& -28,794,164,697,367,780,864 \beta^{8}-1,137,630,004,464,368,357,888 \beta^{7} \\
& +1,201,272,894,249,597,010,176 \beta^{6}+2,920,602,692,653,681,087,776 \beta^{5} \\
& +1,883,711,731,319,280,895,324 \beta^{4}+507,067,392,712,426,159,422 \beta^{3} \\
& +59,630,504,525,663,789,802 \beta^{2}-2,192,824,040,861,997,795 \beta \\
& +17,501,360,748,480,000 .
\end{aligned}
$$

Let lcoeff $(\xi, x)$ be the leading coefficient of $\xi$ with respect to $x$. Then

$$
\operatorname{lcoeff}\left(f_{1}, \delta\right)=-1 \neq 0, \quad \operatorname{lcoeff}\left(r_{12}, \beta\right)=1,781,208,836,997,120,000 \neq 0
$$

Applying Theorem 1 in [4], we have the decomposition

$$
\begin{aligned}
V\left(f_{1}, f_{2}, f_{3}\right)= & V\left(f_{1}, f_{2}, f_{3}, \operatorname{lcoeff}\left(f_{1}, \delta\right)\right) \cup V\left(\frac{f_{1}, f_{2}, f_{3}, r_{12}, r_{13}, \operatorname{lcoeff}\left(r_{12}, \beta\right)}{\operatorname{lcoeff}\left(f_{1}, \delta\right)}\right) \\
& \cup V\left(\frac{f_{1}, f_{2}, f_{3}, r_{12}, r_{13}, r_{23}}{\operatorname{lcoeff}\left(f_{1}, \delta\right), \operatorname{lcoeff}\left(r_{12}, \beta\right)}\right),
\end{aligned}
$$


where $V\left(\frac{\xi_{1}, \xi_{2}, \ldots, \xi_{n}}{\eta_{1}, \eta_{2}, \ldots, \eta_{m}}\right)$ denotes the set of common zeros of $\xi_{i} \mathrm{~S}(i=1,2, \ldots, n)$ at which $\eta_{i} \mathrm{~S}$ $(i=1,2, \ldots, m)$ do not vanish. It follows that

$$
V\left(f_{1}, f_{2}, f_{3}\right)=V\left(f_{1}, f_{2}, f_{3}, r_{12}, r_{13}\right)=V\left(f_{1}, f_{2}, f_{3}, \mathcal{R}_{1}, \mathcal{R}_{2}\right)
$$

Note the resultant

$$
\operatorname{res}\left(\mathcal{R}_{1}, \mathcal{R}_{2}, \beta\right)=c_{0} \neq 0,
$$

where

$c_{0}=5,966,262,075,746,365,907,228,887,461,619,703,253,674,326,002,113,602,564,041,246,673,889,434,463,423,692,800$.

So

$$
V\left(\mathcal{R}_{1}, \mathcal{R}_{2}\right)=\emptyset .
$$

Thus

$$
V\left(f_{1}, f_{2}, f_{3}\right) \cap \mathcal{D}=V\left(f_{1}, f_{2}, f_{3}, \mathcal{R}_{1}, \mathcal{R}_{2}\right) \cap \mathcal{D}=\emptyset .
$$

This completes the proof.

Proof of Theorem 3.1 Since $(\beta, \delta) \in \mathcal{D}$, it follows from (3.8) that the zeros of $L_{i}$ are determined by those of $f_{i}, i=1,2,3$, respectively. By Lemma 3.1, we see that

$$
V\left(L_{1}, L_{3}, L_{3}\right) \cap \mathcal{D}=\emptyset .
$$

Thus, $\left(x_{4}, y_{4}\right)$ is a weak focus of order up to 3 when $(\beta, \delta, \varepsilon) \in \Lambda$.

In what follows, we find the parameter conditions of each order; moreover, we prove that $\left(x_{4}, y_{4}\right)$ is a stable weak focus when it is order 3 . To make the preparation, let

$$
\mathcal{P}:=\left\{(\beta, \delta) \in \mathbb{R}_{2}: \mathcal{R}_{1}=0, \beta \in I_{1}, \delta=-\frac{\chi_{1}(\beta)}{\chi_{2}(\beta)}\right\},
$$

where

$$
\begin{aligned}
I_{1}:= & {\left[\frac{4,560,287}{536,870,912}, \frac{142,509}{16,777,216}\right], } \\
\chi_{1}:= & 2 \beta\left(1024 \beta^{9}+11,648 \beta^{8}+637,440 \beta^{7}+2,535,296 \beta^{6}-3,034,496 \beta^{5}\right. \\
& \left.-252,024 \beta^{4}+64,672 \beta^{3}+3460 \beta^{2}+76 \beta-1\right), \\
\chi_{2}:= & 5120 \beta^{10}+51,712 \beta^{9}+192,000 \beta^{8}+1,575,936 \beta^{7}+6,133,120 \beta^{6} \\
& -1,954,656 \beta^{5}-699,568 \beta^{4}-44,256 \beta^{3}-2460 \beta^{2}-92 \beta+1 .
\end{aligned}
$$


It suffices to prove that $\mathcal{P} \neq \emptyset$ is well defined. By the Maple command "realroot $\left(\chi_{2}, 1 / 10^{6}\right)$ " to isolate the real roots of $\chi_{2}$, we get two consecutive intervals

$$
\left[-\frac{1,002,639}{16,777,216},-\frac{8,021,111}{134,217,728}\right], \quad\left[\frac{4,597,919}{536,870,912}, \frac{143,685}{16,777,216}\right] \text {. }
$$

It is easy to check that $I_{1} \subset\left(0, \frac{4,597,919}{536,870,912}\right)$. Thus $\chi_{2}(\beta) \neq 0$ for all $\beta \in I_{1}$ and $\mathcal{P}$ is well defined. Moreover, using the Maple command "realroot $\left(\mathcal{R}_{1}, 1 / 10^{6}\right)$ ", we get only three intervals: $\left[-\frac{43,210,753}{8,388,608},-\frac{21,099}{4096}\right], I_{1}$, and $I_{2}:=\left[\frac{17,973,673}{8,388,608}, \frac{8,986,837}{4,194,304}\right]$. Thus, $\mathcal{P} \neq \emptyset$ is well defined.

Theorem 3.2 Let $(\beta, \delta, \varepsilon) \in \Lambda$. Then

(i) $\left(x_{4}, y_{4}\right)$ is order 1 if $(\beta, \delta, \varepsilon) \in \Lambda_{1}:=\left\{(\beta, \delta, \varepsilon) \in \Lambda: f_{1}(\beta, \delta) \neq 0\right\}$;

(ii) $\left(x_{4}, y_{4}\right)$ is order 2 if $(\beta, \delta, \varepsilon) \in \Lambda_{2}:=\left\{(\beta, \delta, \varepsilon) \in \Lambda: f_{1}(\beta, \delta)=0,(\beta, \delta) \notin \mathcal{P}\right\}$;

(iii) $\left(x_{4}, y_{4}\right)$ is a stable weak focus with order 3 if $(\beta, \delta, \varepsilon) \in \Lambda_{3}:=\Lambda \backslash\left(\Lambda_{1} \cup \Lambda_{2}\right)$.

The following lemma is useful in the proof of Theorem 3.2.

Lemma 3.2 $V\left(f_{1}, f_{2}\right) \cap \mathcal{D}=\mathcal{P}$.

Proof By Lemma 2 in [4], we have the decomposition

$$
\begin{aligned}
V\left(f_{1}, f_{2}\right) & =V\left(f_{1}, f_{2}, \operatorname{lcoeff}\left(f_{1}, \delta\right)\right) \cup V\left(\frac{f_{1}, f_{2}, r_{12}}{\operatorname{lcoeff}\left(f_{1}, \delta\right)}\right) \\
& =V\left(f_{1}, f_{2}, r_{12}\right) .
\end{aligned}
$$

Thus

$$
V\left(f_{1}, f_{2}\right) \cap \mathcal{D}=V\left(f_{1}, f_{2}, r_{12}\right) \cap \mathcal{D}=V\left(f_{1}, f_{2}, \mathcal{R}_{1}\right) \cap \mathcal{D} .
$$

From the argument just above Theorem 3.2, we see that the positive zeros of $\mathcal{R}_{1}$ are just in the intervals $I_{1}$ and $I_{2}$. Since $I_{2}=\left[\frac{17,973,673}{8,388,608}, \frac{8,986,837}{4,194,304}\right] \subset\left(\frac{1}{2},+\infty\right)$, it can be inferred that

$$
V\left(f_{1}, f_{2}, r_{12}\right) \cap \mathcal{D}=\left\{(\beta, \delta) \in \mathbb{R}_{2}: \beta \in I_{1}, \mathcal{R}_{1}=0, f_{1}=f_{2}=0\right\} \cap \mathcal{D}
$$

Then we further reduce the right-hand side by pseudo-division [17]. To find the dependence of $\delta$ on $\beta$, we employ the Maple command "prem $(\xi, \eta, x)$ " to get the pseudoreminder of $\xi$ divided by $\eta$ and obtain

$$
w_{3}:=\operatorname{prem}\left(w_{1}, w_{2}, \beta\right)=25,480,396,800,000 \beta^{10}(2 \beta+1)^{7} \varphi^{2}(\beta)\left(\chi_{2}(\beta) \delta+\chi_{1}(\beta)\right),
$$

where

$$
w_{1}=\operatorname{prem}\left(f_{2}, f_{1}, \beta\right), \quad w_{2}=\operatorname{prem}\left(f_{1}, w_{1}, \beta\right)
$$

and the expressions are given in the Appendix, and

$$
\varphi=2304 \beta^{6}-2048 \beta^{5}-49,024 \beta^{4}+105,886 \beta^{3}-54,553 \beta^{2}+6716 \beta-122 .
$$


Since $f_{1}=f_{2}=0$, we can deduce that $w_{1}=w_{2}=w_{3}=0$. Moreover, similar to the proof of $\chi_{2}(\beta) \neq 0$ for all $\beta \in I_{1}$ (just below (3.9)), one can easily get that $\varphi(\beta) \neq 0$ for all $\beta \in I_{1}$. Thus, from $w_{3}=0$ it follows that

$$
\delta=-\frac{\chi_{1}(\beta)}{\chi_{2}(\beta)} .
$$

Similar to the proof of $\chi_{2}(\beta) \neq 0$ for all $\beta \in I_{1}$, we can obtain that the derivative $\left(-\frac{\chi_{1}(\beta)}{\chi_{2}(\beta)}\right)^{\prime}>0$ for all $\beta \in I_{1}$. So the function $-\frac{\chi_{1}(\beta)}{\chi_{2}(\beta)}$ is strictly increasing in $I_{1}$, and it can easily be checked that

$$
0.11<-\frac{\chi_{1}(\beta)}{\chi_{2}(\beta)}<0.12<1-\beta, \quad \beta \in I_{1} .
$$

Therefore,

$$
V\left(f_{1}, f_{2}\right) \cap \mathcal{D}=V\left(f_{1}, f_{2}, \mathcal{R}_{1}\right) \cap \mathcal{D}=\mathcal{P}
$$

Proof of Theorem 3.2 (i) It is easy to see that $\Lambda_{1} \neq \emptyset$. In fact, a simple computation yields that at $(\beta, \delta)=(0.1,0.1), \varepsilon_{1}=0.12$ and $f_{1}=-0.0198<0$. So $(0.1,0.1,0.12) \in \Lambda_{1}$ and $\Lambda_{1} \neq \emptyset$. Therefore, $\left(x_{4}, y_{4}\right)$ is a weak focus with order 1 if $(\beta, \varepsilon, \delta) \in \Lambda_{1}$.

(ii) $\left(x_{4}, y_{4}\right)$ is a weak focus of order 2 if $(\beta, \varepsilon, \delta) \in\left\{(\beta, \varepsilon, \delta) \in \Lambda: f_{1}=0, f_{2} \neq 0\right\}$. By Lemma 3.2, $\Lambda_{2}=\left\{(\beta, \delta, \varepsilon) \in \Lambda: f_{1}=0, f_{2} \neq 0\right\}$. Moreover, it suffices to prove that $\Lambda_{2} \neq \emptyset$. In fact, by setting $\beta=0.012$, one can compute $\left.f_{1}\right|_{\delta=0.03}=-0.0000103068$ and $\left.f_{1}\right|_{\delta=0.033}=$ 0.000010142955 . Thus, there is a certain $\delta_{*} \in(0.03,0.033)$ such that

$$
f_{1}\left(0.012, \delta_{*}\right)=0
$$

On the other hand, applying the Maple command "realroot(., $\left.1 / 10^{6}\right)$ " to the polynomial $f_{2}(0.012, \delta)$, we obtain the first two positive intervals $\left[\frac{5,787,933}{134,217,728}, \frac{2,893,967}{67,108,864}\right]$ and $\left[\frac{765,987}{8,388,608}\right.$, $\left.\frac{6,127,897}{67,108,864}\right]$. Since $\frac{5,787,933}{134,217,728} \approx 0.04312346131$, we see that

$$
f_{2}(0.012, \delta) \neq 0, \quad \delta \in(0.03,0.033)
$$

By $(2.1)$, one has $\varepsilon_{*}:=\left.\varepsilon_{1}\right|_{(\varepsilon, \delta)=\left(0.012, \delta_{*}\right)}$. Therefore, $\left(0.012, \delta_{*}, \varepsilon_{*}\right) \in \Lambda_{2}$ and hence $\Lambda_{2} \neq \emptyset$.

(iii) $\left(x_{4}, y_{4}\right)$ is a weak focus of order 3 if $(\beta, \varepsilon, \delta) \in\left\{(\beta, \varepsilon, \delta) \in \Lambda: f_{1}=0, f_{2}=0\right\}$. By Theorem 3.1 and Lemma 3.2, we see that

$$
\Lambda_{3}=\left\{(\beta, \varepsilon, \delta) \in \Lambda:(\beta, \varepsilon) \in \mathcal{P}, \varepsilon=\varepsilon_{1}\right\} .
$$

Then we claim that

$$
f_{3}(\beta, \delta)>0, \quad(\beta, \delta) \in \mathcal{P} .
$$

In fact, substituting $\delta=-\frac{\chi_{1}(\beta)}{\chi_{2}(\beta)}$ into $f_{3}$, we have

$$
\tilde{f}_{3}(\beta):=f_{3}\left(\beta,-\frac{\chi_{1}(\beta)}{\chi_{2}(\beta)}\right) .
$$


With a Maple command, we can easily compute the derivative

$$
\tilde{f}_{3}^{\prime}(\beta)=\frac{v_{1}(\beta)}{v_{2}(\beta)}
$$

where $v_{1}$ and $v_{2}$ are polynomials with order 201 and 190, respectively. We omit the concrete expressions of the two functions. Moreover, by the Maple command "realroot $\left(v_{1}(\beta) v_{2}(\beta), 1 / 10^{6}\right)$ ", we have two consecutive intervals

$$
\left[\frac{1,112,351}{134,217,728}, \frac{4,449,405}{536,870,912}\right], \quad\left[\frac{4,582,613}{536,870,912}, \frac{2,291,307}{268,435,456}\right] .
$$

It is easy to check that

$$
I_{1}=\left[\frac{4,560,287}{536,870,912}, \frac{142,509}{16,777,216}\right] \subset\left(\frac{4,449,405}{536,870,912}, \frac{4,582,613}{536,870,912}\right) .
$$

Hence, $\tilde{f}_{3}^{\prime}(\beta) \neq 0$ for all $\beta \in I_{1}$ and $\tilde{f}_{3}$ is continuous and strictly monotone in $I_{1}$. Since

$$
\tilde{f}_{3}\left(\frac{4,560,287}{536,870,912}\right) \approx 0.001805786293, \quad \tilde{f}_{3}\left(\frac{142,509}{16,777,216}\right) \approx 0.001806068008
$$

it can be deduced that $\tilde{f}_{3}(\beta)>0$ for $\beta \in I_{1}$ and (3.11) holds. Therefore, $\left(x_{4}, y_{4}\right)$ is a stable weak focus of order 3 when $(\beta, \delta, \varepsilon) \in \Lambda_{3}$.

By the classical Hopf bifurcation theorem [5], there are at most three limit cycles bifurcated from a Hopf bifurcation in this system.

\section{Simulation and conclusions}

To display two limit cycles in this system, we set $(\beta, \delta, \varepsilon)=(0.0085,0.112,0.00997691)$. Then $\left(x_{4}, y_{4}\right)=(0.05851163234,0.7709768027)$. In Fig. 1(a) and Fig. 2(a), we plot orbits from $P_{1}\left(0.05855, y_{4}\right)$ and $P_{2}\left(0.05856, y_{4}\right)$, respectively. However, from those two figures, one can hardly see whether the orbits spiral outward or inward. Thus, in Fig. 1(b) and Fig. 2(b), we zoom in the orbits near $P_{1}$ and $P_{2}$, respectively. It shows that the orbit from $P_{1}$ spirals outward, while the orbit from $P_{2}$ spirals inward, as $t \rightarrow+\infty$. Thus, there is a stable limit cycle in the annual regions bounded by the two orbits from $P_{1}$ and $P_{2}$. In Fig. 3, the orbit from $P_{3}\left(0.087, y_{4}\right)$ spirals outward. So there is an unstable limit cycle between the orbits from $P_{2}$ and $P_{3}$.

In this paper, we identify a weak focus of order up to 3 for system (1.3). In [27], Zhu and Lan investigated the saddle-node bifurcation and Hopf bifurcation of codimension 1 in this system. In [8], Gong and Huang studied Bogdanov-Takens bifurcation at the cusp of codimension 2 in this system. Our study supplements the qualitative properties of equilibria in this system, shows that there are at most three limit cycles bifurcated from a Hopf bifurcation. The results in $[8,27]$ and this paper reveal that the codimension of local bifurcations in system (1.3) is at most 3 . 


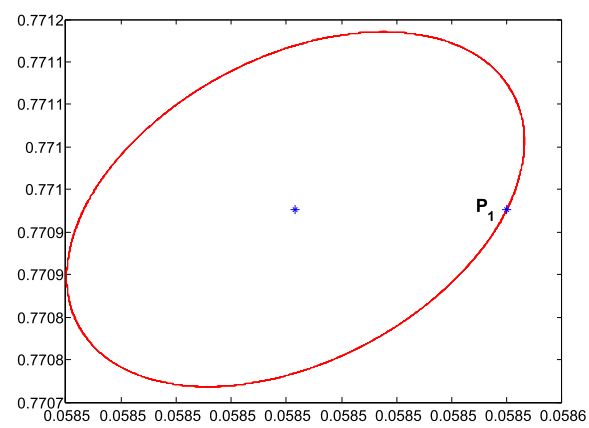

(a)

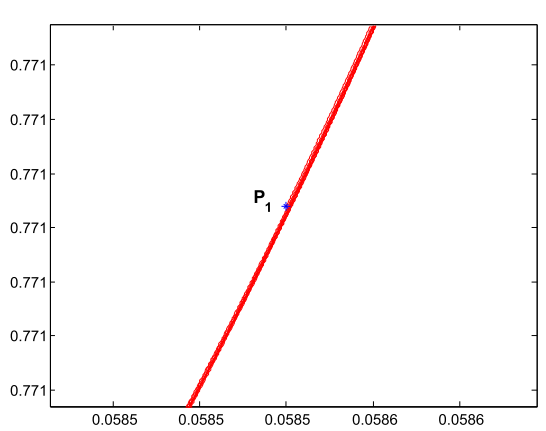

(b)

Figure 1 Orbit from $P_{1}$ spirals outward

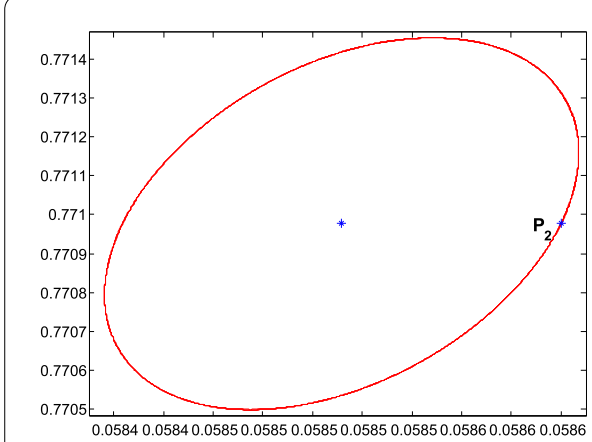

(a)

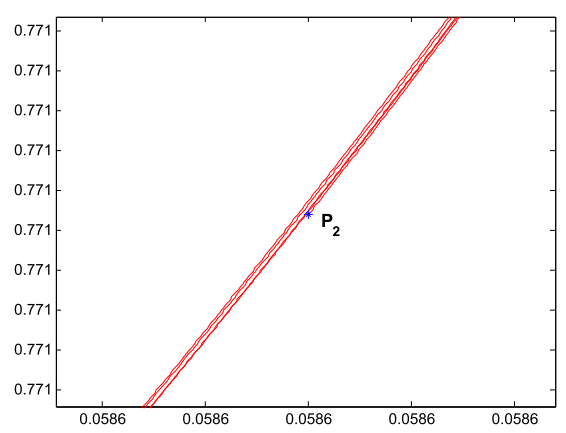

(b)

Figure 2 Orbit from $P_{2}$ spirals inward

Figure 3 Orbit from $P_{3}$ spirals outward

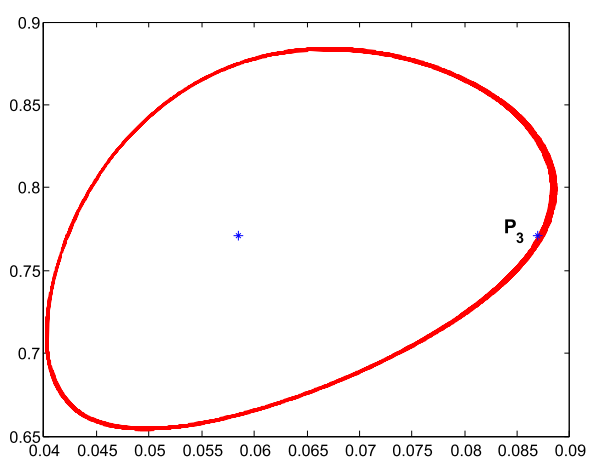

\section{Appendix}

$f_{3}$ in (3.8) is given by

$$
\begin{aligned}
f_{3}= & 96,731,136 \beta^{12} \delta^{6}+721,649,664 \beta^{11} \delta^{7}+2,794,354,688 \beta^{10} \delta^{8}+5,410,942,208 \beta^{9} \delta^{9} \\
& -1,560,727,360 \beta^{8} \delta^{10}-34,792,929,176 \beta^{7} \delta^{11}-88,326,105,106 \beta^{6} \delta^{12} \\
& -120,969,984,391 \beta^{5} \delta^{13}-103,238,957,197 \beta^{4} \delta^{14}-56,414,336,562 \beta^{3} \delta^{15} \\
& -19,214,245,368 \beta^{2} \delta^{16}-3,715,638,831 \beta \delta^{17}-311,347,881 \delta^{18}
\end{aligned}
$$




$$
\begin{aligned}
& -193,462,272 \beta^{12} \delta^{5}-1,515,257,856 \beta^{11} \delta^{6}-5,302,849,536 \beta^{10} \delta^{7} \\
& -5,687,015,424 \beta^{9} \delta^{8}+34,784,497,408 \beta^{8} \delta^{9}+183,262,825,224 \beta^{7} \delta^{10} \\
& +428,364,184,432 \beta^{6} \delta^{11}+596,890,067,742 \beta^{5} \delta^{12}+530,640,116,824 \beta^{4} \delta^{13} \\
& +303,520,979,088 \beta^{3} \delta^{14}+108,038,736,288 \beta^{2} \delta^{15}+21,746,905,794 \beta \delta^{16} \\
& +1,887,281,424 \delta^{17}-96,731,136 \beta^{12} \delta^{4}-329,711,616 \beta^{11} \delta^{5}-357,365,760 \beta^{10} \delta^{6} \\
& -7,943,540,736 \beta^{9} \delta^{7}-76,738,029,696 \beta^{8} \delta^{8}-327,868,403,096 \beta^{7} \delta^{9} \\
& -790,827,616,688 \beta^{6} \delta^{10}-1,180,047,690,204 \beta^{5} \delta^{11}-1,127,224,701,235 \beta^{4} \delta^{12} \\
& -689,017,098,052 \beta^{3} \delta^{13}-259,999,332,192 \beta^{2} \delta^{14}-55,014,678,765 \beta \delta^{15} \\
& -4,977,345,762 \delta^{16}-65,470,464 \beta^{11} \delta^{4}-1,497,653,248 \beta^{10} \delta^{5} \\
& -1,938,664,448 \beta^{9} \delta^{6}+39,046,967,040 \beta^{8} \delta^{7}+244,518,144,840 \beta^{7} \delta^{8} \\
& +710,636,624,720 \beta^{6} \delta^{9}+1,214,492,149,230 \beta^{5} \delta^{10}+1,294,446,982,792 \beta^{4} \delta^{11} \\
& +865,905,700,612 \beta^{3} \delta^{12}+351,842,676,004 \beta^{2} \delta^{13}+790,68,647,724 \beta \delta^{14} \\
& +7,504,928,688 \delta^{15}+28,016,640 \beta^{11} \delta^{3}+1,160,488,960 \beta^{10} \delta^{4} \\
& +6,104,721,920 \beta^{9} \delta^{5}+1,348,262,016 \beta^{8} \delta^{6}-82,124,328,304 \beta^{7} \delta^{7} \\
& -343,639,174,498 \beta^{6} \delta^{8}-718,676,593,199 \beta^{5} \delta^{9}-887,593,367,266 \beta^{4} \delta^{10} \\
& -665,765,134,544 \beta^{3} \delta^{11}-295,896,637,096 \beta^{2} \delta^{12}-71,325,295,217 \beta \delta^{13} \\
& -7,140,536,213 \delta^{14}+237,187,072 \beta^{10} \delta^{3}-734,119,936 \beta^{9} \delta^{4}-3,071,971,584 \beta^{8} \delta^{5} \\
& +13,284,843,280 \beta^{7} \delta^{6}+95,475,893,600 \beta^{6} \delta^{7}+256,301,099,196 \beta^{5} \delta^{8} \\
& +379,247,745,456 \beta^{4} \delta^{9}+326,564,669,496 \beta^{3} \delta^{10}+161,106,471,060 \beta^{2} \delta^{11} \\
& +42,020,446,450 \beta \delta^{12}+4,451,967,104 \delta^{13}-226,289,664 \beta^{10} \delta^{2}-752,545,792 \beta^{9} \delta^{3} \\
& +142,250,752 \beta^{8} \delta^{4}-556,322,736 \beta^{7} \delta^{5}-15,433,168,944 \beta^{6} \delta^{6} \\
& -55,876,146,576 \beta^{5} \delta^{7}-102,248,964,518 \beta^{4} \delta^{8}-103,412,295,132 \beta^{3} \delta^{9} \\
& -57,273,221,944 \beta^{2} \delta^{10}-16,256,443,195 \beta \delta^{11}-1,820,911,280 \delta^{12} \\
& +383,757,312 \beta^{9} \delta^{2}+590,915,840 \beta^{8} \delta^{3}-25,879,728 \beta^{7} \delta^{4}+1,235,251,616 \beta^{6} \delta^{5} \\
& +7,222,295,148 \beta^{5} \delta^{6}+17,288,566,128 \beta^{4} \delta^{7}+21,099,650,344 \beta^{3} \delta^{8} \\
& +13,099,102,924 \beta^{2} \delta^{9}+4,031,542,872 \beta \delta^{10}+471,592,872 \delta^{11}-163,747,584 \beta^{9} \delta \\
& -613,492,032 \beta^{8} \delta^{2}-654,952,440 \beta^{7} \delta^{3}-431,442,486 \beta^{6} \delta^{4}-529,750,101 \beta^{5} \delta^{5} \\
& -1,697,582,593 \beta^{4} \delta^{6}-2,779,581,518 \beta^{3} \delta^{7}-1,863,751,104 \beta^{2} \delta^{8} \\
& -602,714,576 \beta \delta^{9}-70,272,664 \delta^{10}+106,369,536 \beta^{8} \delta+218,433,512 \beta^{7} \delta^{2} \\
& +246,572,592 \beta^{6} \delta^{3}+61,714,598 \beta^{5} \delta^{4}-10,586,824 \beta^{4} \delta^{5}+222,984,680 \beta^{3} \delta^{6} \\
& +153,830,940 \beta^{2} \delta^{7}+47,504,424 \beta \delta^{8}+4,596,480 \delta^{9}-20,280,960 \beta^{8} \\
& -44,424,632 \beta^{7} \delta-93,660,320 \beta^{6} \delta^{2}-77,282,164 \beta^{5} \delta^{3}+17,502,537 \beta^{4} \delta^{4}
\end{aligned}
$$




$$
\begin{aligned}
& -8,688,656 \beta^{3} \delta^{5}-6,098,760 \beta^{2} \delta^{6}-1,410,248 \beta \delta^{7}-5,197,976 \beta^{7}-1,721,328 \beta^{6} \delta \\
& +13,698,342 \delta^{2} \beta^{5}-831,544 \beta^{4} \delta^{3}+21,924 \beta^{3} \delta^{4}+1,250,858 \beta^{6}-625,429 \beta^{5} \delta .
\end{aligned}
$$

$w_{1}$ and $w_{2}$ in (3.10) are given by

$$
\begin{aligned}
w_{1}= & 480 \beta^{2}\left(2304 \beta^{7} \delta^{2}+2304 \beta^{6} \delta^{3}-4608 \beta^{7} \delta-8192 \beta^{6} \delta^{2}-2048 \beta^{5} \delta^{3}-2304 \beta^{7}\right. \\
& -2048 \beta^{6} \delta-51,072 \beta^{5} \delta^{2}-49,024 \beta^{4} \delta^{3}-1024 \beta^{6}+86,272 \beta^{5} \delta+224,196 \beta^{4} \delta^{2} \\
& +105,886 \beta^{3} \delta^{3}+43,904 \beta^{5}-121,096 \beta^{4} \delta-220,298 \beta^{3} \delta^{2}-54,553 \beta^{2} \delta^{3} \\
& -58,308 \beta^{4}+49,394 \beta^{3} \delta+58,488 \beta^{2} \delta^{2}+6716 \beta \delta^{3}+16,614 \beta^{3}-8265 \beta^{2} \delta \\
& \left.-3381 \beta \delta^{2}-122 \delta^{3}-856 \beta^{2}+408 \beta \delta+18 \delta^{2}+2 \beta-\delta\right), \\
w_{2}= & -230,400 \beta^{4}\left(512 \beta^{11} \delta^{2}+2,948,096 \beta^{11} \delta+2,949,376 \beta^{10} \delta^{2}+1,179,136 \beta^{11}\right. \\
& +24,999,936 \beta^{10} \delta+25,775,360 \beta^{9} \delta^{2}+10,795,776 \beta^{10}+65,688,320 \beta^{9} \delta \\
& +72,043,136 \beta^{8} \delta^{2}+30,435,072 \beta^{9}+87,579,904 \beta^{8} \delta+104,710,944 \beta^{7} \delta^{2} \\
& +38,775,424 \beta^{8}+55,586,624 \beta^{7} \delta+84,918,096 \beta^{6} \delta^{2}+40,341,024 \beta^{7} \\
& +31,649,056 \beta^{6} \delta+48,360,064 \beta^{5} \delta^{2}+15,650,960 \beta^{6}+2,267,712 \beta^{5} \delta \\
& +15,585,152 \beta^{4} \delta^{2}+7,090,912 \beta^{5}+64,832 \beta^{4} \delta+3,417,384 \beta^{3} \delta^{2} \\
& +1,218,464 \beta^{4}-366,024 \beta^{3} \delta+405,096 \beta^{2} \delta^{2}+150,620 \beta^{3}-62,822 \beta^{2} \delta \\
& \left.+21,884 \beta \delta^{2}+7354 \beta^{2}-3793 \beta \delta+202 \delta^{2}+36 \beta-18 \delta\right) .
\end{aligned}
$$

\section{Acknowledgements}

The author would like to thank the editors and reviewers sincerely.

\section{Funding}

The author is supported financially by the Foundation of Sichuan Education Committee (18ZB0094) and the Program of Chengdu Normal University (2018JY37).

\section{Availability of data and materials}

Not applicable.

\section{Competing interests}

The author declares that there are no competing interests.

\section{Authors' contributions}

The author read and approved the final manuscript.

\section{Publisher's Note}

Springer Nature remains neutral with regard to jurisdictional claims in published maps and institutional affiliations.

Received: 4 May 2019 Accepted: 9 August 2019 Published online: 28 August 2019

\section{References}

1. Beddington, J., Cooke, J.: Harvesting from a prey-predator complex. Ecol. Model. 14, 155-177 (1982)

2. Brauer, F., Castillo-Chavez, C.: Mathematical Models in Population Biology and Epidemiology, 2nd edn. Springer, NewYork (2012)

3. Chen, J., Huang, J., Ruan, S., Wang, J.: Bifurcations of invariant tori in predator-prey models with seasonal prey harvesting. SIAM J. Appl. Math. 73, 1876-1905 (2013)

4. Chen, X., Zhang, W.: Decomposition of algebraic sets and applications to weak centers of cubic systems. J. Comput. Appl. Math. 23, 565-581 (2009) 
5. Chow, S.N., Li, C., Wang, D.: Normal Forms and Bifurcation of Planar Vector Fields. Cambridge University Press, New York (1994)

6. Collins, G., Akritas, A.: Polynomial real root isolation using Descartes rule of signs. In: Proceedings of the 1976 ACM Symposium on Symbolic and Algebraic Computation, pp. 272-275. ACM, New York (1976)

7. Etoua, R., Rousseau, C.: Bifurcation analysis of a generalized Gause model with prey harvesting and a generalized Holling response function of type III. J. Differ. Equ. 249, 2316-2356 (2010)

8. Gong, Y., Huang, J.: Bogdanov-Takens bifurcation in a Leslie-Gower predator-prey model with prey harvesting. Acta Math. Appl. Sin. Engl. Ser. 30, 239-244 (2014)

9. Han, M.: Bifurcation Theory of Limit Cycles. Science Press, Beijing (2013)

10. Hsu, S., Huang, T.: Global stability for a class of predator-prey systems. SIAM J. Appl. Math. 55, 763-783 (1995)

11. Hsu, S., Huang, T.: Uniqueness of limit cycles for a predator-prey system of Holling and Leslie type. Can. Appl. Math. Q. 6, 91-117 (1998)

12. Hsu, S., Huang, T.: Hopf bifurcation analysis for a predator-prey system of Holling and Leslie type. Taiwan. J. Math. 3, 35-53 (1999)

13. Huang, J., Gong, Y., Ruan, S.: Bifurcation analysis in a predator-prey model with constant-yield predator harvesting. Discrete Contin. Dyn. Syst., Ser. B 18, 2101-2121 (2013)

14. Huang, J., Liu, S., Ruan, S., Zhang, X.: Bogdanov-Takens bifurcation of codimension 3 in a predator-prey model with constant-yield predator harvesting. Commun. Pure Appl. Anal. 15, 1053-1067 (2016)

15. Huang, J., Ruan, S., Song, J.: Bifurcations in a predator-prey system of Leslie type with generalized Holling type III functional response. J. Differ. Equ. 257, 1721-1752 (2014)

16. Huang, J., Xia, X., Zhang, X., Ruan, S.: Bifurcation of codimension 3 in a predator-prey system of Leslie type with simplified Holling type IV functional response. Int. J. Bifurc. Chaos 26, 1650034 (2016)

17. Knuth, D.: The Art of Computer Programming, Vol. 2, Seminumerical Algorithms. Addison-Wesley, Reading (1969)

18. Leslie, P.: Some further notes on the use of matrices in population mathematics. Biometrika 35, 213-245 (1948)

19. Leslie, P., Gower, J.: The properties of a stochastic model for the predator-prey type of interaction between two species. Biometrika 47, 219-234 (1960)

20. Li, Y., Xiao, D.: Bifurcations of a predator-prey system of Holling and Leslie types. Chaos Solitons Fractals 34, 606-620 (2007)

21. May, R.: Stability and Complexity in Model Ecosystems. Princeton University Press, Princeton (1973)

22. Murray, J.: Mathematical Biology. Springer, Berlin (1989)

23. Ritt, J.: Differential Algebra. Am. Math. Soc., Providence (1950)

24. Xiao, D., Jennings, L.: Bifurcations of a ratio-dependent predator-prey system with constant rate harvesting. SIAM J. Appl. Math. 65, 737-753 (2005)

25. Xiao, D., Li, W., Han, M.: Dynamics in a ratio-dependent predator-prey model with predator harvesting. J. Math. Anal. Appl. 324, 14-29 (2006)

26. Zhang, Z., Ding, T., Huang, W., Dong, Z.: Qualitative Theory of Differential Equations. Am. Math. Soc., Providence (1992)

27. Zhu, C., Lan, K.: Phase portraits, Hopf bifurcations and limit cycles of Leslie-Gower predator-prey systems with harvesting rates. Discrete Contin. Dyn. Syst., Ser. B 14, 289-306 (2010)

\section{Submit your manuscript to a SpringerOpen ${ }^{\circ}$ journal and benefit from:}

- Convenient online submission

- Rigorous peer review

- Open access: articles freely available online

- High visibility within the field

Retaining the copyright to your article

Submit your next manuscript at $\boldsymbol{~ s p r i n g e r o p e n . c o m ~}$ 